\title{
Effects of Docosahexaenoic Acid Supplementation on Blood Pressure, Heart Rate, and Serum Lipids in Scottish Men with Hypertension and Hypercholesterolemia
}

\author{
Miki Sagara, ${ }^{1,2}$ Marina Njelekela, ${ }^{3}$ Takanori Teramoto, ${ }^{2}$ Takashi Taguchi, ${ }^{2,4}$ Mari Mori, \\ Lesley Armitage, ${ }^{5}$ Nina Birt, ${ }^{6}$ Christopher Birt, ${ }^{7}$ and Yukio Yamori ${ }^{1,2,4}$ \\ ${ }^{1}$ Laboratory of Preventive Nutritional Medicine, Research Institute for Production Development, Kyoto 606-0805, Japan \\ ${ }^{2}$ International Center for Research on Primary Prevention of Cardiovascular Diseases, Kyoto 606-8413, Japan \\ ${ }^{3}$ Department of Physiology, School of Medicine, Muhimbili University of Health and Allied Sciences, P.O. Box 65001, \\ Dar es Salaam, Tanzania \\ ${ }^{4}$ Institute for World Health Development, Mukogawa Women's University, Nishinomiya 663-8143, Japan \\ ${ }^{5}$ Department of Public Health, University of Birmingham, Birmingham B75 2TT, UK \\ ${ }^{6}$ Peninsula Health, Wirral CH41 8DB, UK \\ ${ }^{7}$ Division of Public Health, University of Liverpool, Liverpool L69 2GB, UK
}

Correspondence should be addressed to Yukio Yamori, yamori@cardiacstudy.com

Received 1 November 2010; Accepted 17 January 2011

Academic Editor: C. Borghi

Copyright ( $) 2011$ Miki Sagara et al. This is an open access article distributed under the Creative Commons Attribution License, which permits unrestricted use, distribution, and reproduction in any medium, provided the original work is properly cited.

To investigate the effects of daily supplementation with docosahexaenoic acid (DHA) on coronary heart disease risks in 38 middle-aged men with hypertension and/or hypercholesterolemia in Scotland, a five-week double-blind placebo-controlled dietary supplementation with either $2 \mathrm{~g}$ of DHA or active placebo ( $1 \mathrm{~g}$ of olive oil) was conducted. Percent composition of DHA in plasma phospholipids increased significantly in DHA group. Systolic and diastolic blood pressure and heart rate decreased significantly in DHA group, but not in placebo group. High-density lipoprotein cholesterol (HDL-C) increased significantly, and total cholesterol (TC)/HDL-C and non-HDL-C/HDL-C ratios decreased significantly in both groups. There was no change in TC and non-HDLC. We conclude that $2 \mathrm{~g} /$ day of DHA supplementation reduced coronary heart disease risk factor level improving blood pressure, heart rate, and lipid profiles in hypertensive, hypercholesterolemic Scottish men who do not eat fish on a regular basis.

\section{Introduction}

In Scotland, coronary heart disease (CHD) has been one of the leading causes of death, and standardized mortality rates (SDRs) from CHD have been among the highest in Europe for decades although it has been declining since the late 1970s [1, 2]. Evidence from our epidemiological studies showed an inverse relation between death from $\mathrm{CHD}$ and fish consumption and that fish consumption in Scotland was at the lowest level among 24-study populations in the world $[3,4]$. Diets high in saturated fats, low in fibre, antioxidants, and polyunsaturated fatty acids (PUFAs) were shown to contribute to the higher mortality rates for CHD in Scotland [5]. There have been various public health projects undertaken to facilitate dietary modifications as primary means of the prevention of CHD among the Scottish people. However, dietary efforts to improve CHD risk in Scotland need as well to emphasize the importance of increasing intake of fish and fish products among the general population. Sections of populations, which consume large amounts of marine foods, have a low prevalence of cardiovascular diseases (CVD) [6, 7]. Our epidemiological study indicated plasma docosahexaenoic acid (DHA) level as a useful biomarker of the frequency of fish intake [8]. The intake of fish oils has been associated with a significant reduction in blood pressure (BP) [9], triglycerides, and verylow density lipoprotein cholesterol [10]. The effect of fish oil on reducing heart rate (HR), a CVD risk factor, has also been 
recognized [11]. We further confirmed a significant inverse association of $\omega-3$ PUFAs and PUFAs to saturated fatty acids (FAs) ratio with CHD mortality in 25 population samples drawn in 16 countries [12].

We hypothesize that frequent intake of fish or fish products may reduce CHD risk factors among people who eat little or no fish in Scotland. Therefore, the objective of this study was to find out if dietary long chain PUFAs and docosahexaenoic acid (DHA) was effective in improving BP, HR, and lipid profile in hypertensive and hypercholesterolemic Scottish men.

\section{Materials and Methods}

2.1. Subjects. Study volunteers were recruited in Spring 2001 in the island of Lewis and Harris in the Western Isles, in the north western part of Scotland. A total of 156 male inhabitants aged 45-59 years from the two areas were randomly selected using a stratified approach. First 12 general practitioners were randomly selected from all the general practitioners on the Isles of Lewis and Harris. Then, using the age-sex registers of the practices within which the $12 \mathrm{GP}$ principals worked, males within the specified age range were randomly selected. The sampling was proportionate to the numbers on each GP list to prevent geographical bias. Exclusion criteria included severe hypertension ( $\mathrm{SBP} \geq 180 \mathrm{mmHg}$ and/or DBP $\geq 110 \mathrm{mmHg}$ ), the presence of diabetes mellitus and any other chronic illness that could affect BP or blood lipid concentration or limit the individual's ability to participate in the study, and the use of antihypertensive drugs, cholesterol-lowering drugs, and any medication known to affect BP or lipid concentrations. Subjects who withdrew during the intervention period or who failed to collect data completely were excluded from the analysis. The Research Ethics Committee of the Western Isles Health Board approved the study design. Each participant signed an informed consent form.

2.2. Study Design. The study was a randomized double-blind placebo-controlled trial involving 156 male inhabitants aged 45-59 in the Isles of Lewis and Harris. The study was conducted in two separate stages. Stage 1 was a screening survey for high-risk subjects, involving a structured questionnaire to obtain information about demographic characteristics, medical history and medication, and a clinical evaluation of height and weight, BP, HR, and fasting blood lipid profile. $14 \mathrm{~mL}$ samples of overnight fasting blood were collected and used as baseline data. Blood lipid profile was measured with a compact, instant blood lipid analyzer. Three BP and HR measurements were taken with an automatic digital BP monitor (HEM-970, OMRON, Kyoto, Japan) after about ten minutes of rest. The average of the three readings is used in this analysis as baseline data. Weight and height were measured with participants wearing light clothing and without shoes. Weight was measured to the nearest kilogram using a balance scale. Height was measured to the nearest centimetre using a wall-mounted ruler. A followup examination was conducted after five weeks of dietary supplementation.

112 men who were hypertensive (systolic BP (SBP) $\geq 130 \mathrm{mmHg}$ ) and/or hypercholesterolemic (total cholesterol (TC) $\geq 220 \mathrm{mg} / \mathrm{dL}$ or $5.7 \mathrm{mmol} / \mathrm{L}$ ) were eligible to enter stage 2 of 5-week dietary supplementation, which was originally a 4-armed trial [13, 14]. Of these four arms, only those two involving DHA-containing diets and active placebo diets were relevant as subjects of this report; 25 and 31 participants were assigned to the DHA group and to the active placebo group, respectively, randomly in a double-blind fashion. In the DHA group, DHA powder containing calcium encapsulated DHA (2 g/day) mixed in bread rolls were consumed in addition to the usual diet. The active placebo group received bread rolls containing calciumencapsulated olive oil powder ( $1 \mathrm{~g} /$ day). Both powders were supplied from KITII Co. Tokyo, Japan. The bread rolls were made by a local baker on Lewis and were identical with respect to shape, colour, and packaging. Their contents are shown in Table 1. Fresh rolls were supplied on alternate days with extra quantities provided when necessary to accommodate weekends. The fact that the DHA powder was calcium encapsulated made it resistant to further oxidation and this also reduced the smell of fish oil in the food. The subjects were asked to keep the bread rolls in their refrigerators until the time of consumption. They agreed to maintain a stable body weight throughout the study and to refrain from any significant change in physical activity.

Prior to commencement of the dietary intervention, the participants were given detailed written and verbal information about the intervention and the supplements. They were provided with food record forms and were asked to record their intervention consumption daily. Two members of the research team visited the participants on two more occasions during the study period, at weeks 2 and 4, respectively. During each visit, dietary counselling, adverse events, compliance assessments, and any medication changes were documented.

At the beginning of the sixth week, the participants were invited for the final assessment. BP, HR, height, and weight measurements were repeated as at the preliminary screening, and fasting blood samples were collected. Participants completed a one-page questionnaire intended to identify any adverse effects of the supplements and they were asked to return the food record forms.

2.3. Laboratory Analyses. The serum and plasma samples were collected into evacuated tubes (Vacutainer, Wako Company, Kyoto, Japan), centrifuged at $3000 \mathrm{rpm}$ for 10 minutes at $23^{\circ} \mathrm{C}$, and stored at $-20^{\circ} \mathrm{C}$ and at $-70^{\circ} \mathrm{C}$, respectively. All samples were shipped to the Central Laboratory for WHO-CARDIAC Study in Kyoto, Japan for the standardized analyses [15]. Stable conditions $\left(-70^{\circ} \mathrm{C}\right)$ were maintained during transportation to Kyoto. TC and HDL-C were measured using an Express-Plus Operator Machine, (Ciba Corning Diagnostics Corp. West Lake Village USA). NonHDL-C was calculated from the difference between TC and 
TABLE 1: Contents of bread rolls.

\begin{tabular}{lcc}
\hline Nutrients & Placebo & DHA \\
\hline Energy (kcal) & 251.7 & 249 \\
Protein (g) & 9.1 & 8.7 \\
Fat (g) & 8.5 & 10.6 \\
Carbohydrate (g) & 33.3 & 28.7 \\
Dietary fibre (g) & 1.6 & 1.5 \\
Sodium (mg) & 317.5 & 301 \\
Olive oil (g) & 1.0 & 0.0 \\
Docosahexaenoic acid (g) & 0.0 & 2.0 \\
\hline
\end{tabular}

Placebo: Olive oil-containing bread rolls; DHA: docosahexaenoic acid(DHA-)containing bread rolls

HDL-C: (Non-HDL-C = TC - HDL-C) [16]. Serum TC was also analyzed on site to detect high risk subjects. These data were used only for this purpose.

The total lipids were extracted from $500 \mu \mathrm{L}$ of plasma according to the method of Folch et al. [17]. Heptadecanoic acid (H-4515 Sigma Chemical Company, St. Louis, Missouri) was added as an internal standard, chloroform-methanol $(2: 1 \mathrm{v} / \mathrm{v})$ was added as a solvent, and butylated hydroxytoluene (B-1378 Sigma Chemical Company, St. Louis, Missouri) was added as an antioxidant. Total phospholipids were separated by solid-phase extraction using silica gel columns (237-0075 Wakogel C-200 (Silica Gel), Wako Pure Chemicals), kept overnight in methanol (137-01823 Methanol, Wako Pure Chemicals), and later evaporated to dryness. Transmethylation was done using TCI-SU3 (trifluoromethyl phenyl-trimethylammonium hydroxide) (5\% in methanol) (T-0961, Tokyo Kasei Kogyo Co. Ltd, Tokyo, Japan) and methanol $(1: 1 \mathrm{v} / \mathrm{v})$. FA methyl esters were measured using a gas-liquid chromatograph (Shimadzu GC14B, Shimadzu Co. Kyoto, Japan). Retention times for each FA were determined using standards obtained from Sigma Chemicals (Sigma Chemical Co., St. Louis, Missouri). The results were integrated on a Shimadzu C-R4A integrator, and the FA concentrations were reported as percentage composition of FA in plasma phospholipids.

2.4. Statistical Analysis. Statistical analyses were performed using SPSS 15.0 for Windows (SPSS Japan Inc., Tokyo, Japan). The analysis was carried out on an "as treated" basis in order to estimate the effect of DHA itself. Baseline values for serum lipids and anthropometric measurements were taken as the measurements obtained at the screening process. Because FA, HDL cholesterol, and the food consumption over the intervention period were not normally distributed, Wilcoxon rank sum tests were applied for the between group comparisons and Wilcoxon signed-rank tests were applied for the within group comparisons as for those parameters. As for the other parameters that were normally distributed, the between group differences were tested by Student's t-tests and the within group differences were tested by paired Student's t-tests. Data are expressed as mean \pm SD if not otherwise specified. $P$ values $<.05$ were considered significant.
TABLE 2: Subjects characteristics at baseline.

\begin{tabular}{lccc}
\hline & $\begin{array}{c}\text { Placebo } \\
(n=23)\end{array}$ & $\begin{array}{c}\text { DHA } \\
(n=15)\end{array}$ & $\begin{array}{c}P \\
\text { values* }\end{array}$ \\
\hline Age $(\mathrm{y})$ & $52.3 \pm 4.4$ & $52.7 \pm 3.2$ & .7728 \\
Height $(\mathrm{em})$ & $175.6 \pm 6.7$ & $176.9 \pm 6.1$ & .5308 \\
Weight $(\mathrm{Kg})$ & $83.7 \pm 11.8$ & $82.7 \pm 12.3$ & .8009 \\
$\begin{array}{l}\text { Body mass index } \\
\left(\mathrm{kg} / \mathrm{m}^{2}\right)\end{array}$ & $27.1 \pm 3.1$ & $26.4 \pm 3.3$ & .4869 \\
\hline
\end{tabular}

Values are presented as mean \pm SD. DHA: docosahexaenoic acid.

${ }^{*} P$ values between the placebo and DHA group.

\section{Results and Discussion}

3.1. Results. Out of 56 eligible participants, 10 withdrew during the 5-week intervention and 46 subjects returned for the follow-up examination. Prior to the analysis, 3 participants were excluded because of missing data, and 5 more who had not consumed at least $80 \%$ of the total amount of bread rolls according to the food records were excluded for noncompliance with the diet. Therefore, the data from 38 subjects were used for analysis, and the number of the participants in the DHA and the active placebo group was 15 and 23, respectively. There were no significant differences in the average rate of the consumption of bread rolls between the DHA group and the active placebo group $(92.6 \pm 6.9 \%$ versus $94.8 \pm 5.2 \%$, resp., $P=.306)$. The baseline characteristics of the subjects assigned to the DHA and active placebo olive oil are shown in Table 2. There was no significant difference in age, height, weight, and body mass index between the two groups.

The effects of DHA supplementation on plasma $\omega-3$ and $\omega-6$ PUFAs are shown in Table 3 . Owing to a maintenance failure, some of the plasma samples were not kept at the correct temperature of $-70^{\circ} \mathrm{C}$ for a period, so that the number of blood samples available for the measurement of FAs was limited to 34 subjects from placebo and DHA groups. Percentage composition of DHA in plasma phospholipids increased significantly, more than 3 -fold in the DHA group $(P<.001)$. When PUFAs levels were analysed in terms of total $\omega-3$ and $\omega-6$ PUFAs percent composition in plasma phospholipids, a significant increase was observed in the total $\omega$-3 PUFAs in the DHA group $(P<.01)$. Similarly, in the placebo group receiving olive oil, a significant increase in the total $\omega-6$ PUFAs was observed $(P<.01)$. There were significant between group differences in the change in DHA and total $\omega$-3 PUFAs.

Table 4 shows the body weight, HR, BP and lipid profiles at baseline and after intervention in the placebo and DHA groups. There was a significant reduction in mean SBP $(P<.001)$, DBP $(P<.01)$, and HR $(P<.05)$ in DHA supplemented group but not in the placebo group. The analysis of serum lipid profiles showed an increase in HDL-C and decrease in TC/HDL-C and non-HDL-C/HDL$\mathrm{C}$ ratios both in the placebo and DHA groups. There was no significant change in the TC and non-HDL-C during the 5 weeks of intervention in either group. 
TABLE 3: $\omega-3$ and $\omega-6$ fatty acids in plasma phospholipids at baseline and at 5 weeks and change from the baseline.

\begin{tabular}{lcccccc}
\hline \multirow{2}{*}{ Fatty acid } & \multicolumn{2}{c}{ Placebo $(n=20)$} & \multicolumn{3}{c}{ DHA $(n=14)$} \\
& Baseline & 5 Weeks & Change (5-weeks-baseline) & Baseline & 5 Weeks & Change (5-weeks-baseline) \\
\hline EPA (C20:5) (\%) & $1.50 \pm 1.13$ & $1.53 \pm 0.79$ & $0.04 \pm 1.09$ & $1.24 \pm 1.30$ & $1.65 \pm 1.20$ & $0.41 \pm 1.13$ \\
DHA (C22:6) (\%) & $1.58 \pm 0.64$ & $1.47 \pm 0.64$ & $-0.11 \pm 0.78$ & $1.04 \pm 0.47$ & $3.40 \pm 0.90^{* * *}$ & $2.36 \pm 0.79^{\dagger \dagger \dagger}$ \\
PUFAs $\omega$-3 series (\%) & $3.08 \pm 1.54$ & $3.00 \pm 1.14$ & $-0.07 \pm 1.41$ & $2.28 \pm 1.61$ & $5.05 \pm 1.86^{* *}$ & $2.77 \pm 1.64^{\dagger \dagger \dagger}$ \\
PUFAs $\omega$-6 series (\%) & $16.0 \pm 5.8$ & $19.3 \pm 5.1^{* *}$ & $3.38 \pm 4.45$ & $17.1 \pm 7.0$ & $18.3 \pm 4.3$ & $1.29 \pm 5.64$ \\
$\omega$-3/ $\omega$-6 Ratio & $0.24 \pm 0.18$ & $0.17 \pm 0.09^{*}$ & $-0.07 \pm 0.14$ & $0.15 \pm 0.13$ & $0.30 \pm 0.18^{* *}$ & $0.15 \pm 0.14^{\dagger+\dagger}$ \\
\hline
\end{tabular}

EPA: eicosapentaenoic acid, DHA: docosahexaenoic acid, PUFAs: polyunsaturated fatty acids.

PUFAs $\omega$-3 series: EPA + DHA; PUFAs $\omega$-6 series: arachidonic acid + linoleic acid.

Placebo: Olive oil-containing bread rolls, DHA: DHA-containing bread rolls. Values are presented as mean \pm SD.

${ }^{*} P<.05,{ }^{* *} P<.01$, and ${ }^{* * *} P<.001$ compared with the baseline. ${ }^{\dagger \dagger \dagger} P<.001$ compared with the placebo group.

TABLE 4: Body weight, heart rate, blood pressure, and lipid profiles in the two groups at baseline and at 5 weeks.

\begin{tabular}{|c|c|c|c|c|}
\hline \multirow{2}{*}{ Parameter } & \multicolumn{2}{|c|}{ Placebo $(n=23)$} & \multicolumn{2}{|c|}{ DHA $(n=15)$} \\
\hline & Baseline & 5 Weeks & Baseline & 5 Weeks \\
\hline Weight (kg) & $83.7 \pm 11.8$ & $83.3 \pm 11.4$ & $82.7 \pm 12.3$ & $83.1 \pm 12.3$ \\
\hline Heart rate (bpm) & $70.7 \pm 14.4$ & $67.0 \pm 10.3$ & $70.4 \pm 9.8$ & $64.8 \pm 9.2^{*}$ \\
\hline Systolic BP (mmHg) & $132.7 \pm 15.9$ & $128.7 \pm 16.3$ & $141.4 \pm 13.5$ & $133.0 \pm 12.9^{* * *}$ \\
\hline Diastolic BP (mmHg) & $80.3 \pm 10.5$ & $77.9 \pm 9.7$ & $86.7 \pm 10.4$ & $81.9 \pm 9.3^{* *}$ \\
\hline $\mathrm{TC}(\mathrm{mg} / \mathrm{dL})$ & $228.9 \pm 36.6$ & $226.4 \pm 36.6$ & $253.6 \pm 45.9$ & $247.7 \pm 40.5$ \\
\hline HDL-C (mg/dL) & $54.8 \pm 12.7$ & $59.3 \pm 11.1^{* *}$ & $55.5 \pm 17.3$ & $64.1 \pm 20.4^{* *}$ \\
\hline Non- HDL-C (mg/dL) & $174.1 \pm 39.8$ & $167.2 \pm 36.6$ & $198.1 \pm 48.7$ & $183.6 \pm 42.0$ \\
\hline TC/HDL-C & $4.4 \pm 1.2$ & $3.9 \pm 0.8^{* *}$ & $4.8 \pm 1.3$ & $4.1 \pm 0.9^{* *}$ \\
\hline Non-HDL-C/HDL-C & $3.4 \pm 1.2$ & $2.9 \pm 0.8^{* *}$ & $3.8 \pm 1.3$ & $3.1 \pm 0.9^{* *}$ \\
\hline
\end{tabular}

Placebo: Olive oil-containing bread rolls, DHA: DHA-containing bread rolls. Values are presented as mean \pm SD. ${ }^{*} P<.05,{ }^{* *} P<.01$, and ${ }^{* * *} P<.001$ compared with the baselines.

Figure 1 shows the changes in SBP, DBP, and HR between baseline and after the intervention. In the DHA group, there were significant reductions in SBP, DBP, and HR. However, there were no significant differences between the two groups in SBP (DHA group; $-8.3 \mathrm{mmHg}$ versus placebo group; $-4.9 \mathrm{mmHg}, P=.19$ ), DBP (DHA group; $-4.1 \mathrm{mmHg}$ versus placebo group; $-2.3 \mathrm{mmHg}, P=.38$ ), and $\mathrm{HR}$ (DHA group; $-5.6 \mathrm{bpm}$ versus placebo group; $-3.7 \mathrm{bpm}, P=.62$ ).

3.2. Discussion. This study evaluated the beneficial effects of supplementation with DHA on BP, HR, and serum lipids in hypertensive and/or hypercholesterolaemic Scottish men. DHA significantly reduced $\mathrm{BP}$ and $\mathrm{HR}$ in the intervention group. HDL-C level was significantly increased and TC/HDL-C and non-HDL-C/HDL-C ratios were therefore significantly decreased both in the DHA supplemented as well as in the placebo groups.

We demonstrated a $5.8 \% \quad(8.3 \mathrm{mmHg})$ and $3.7 \%$ $(4.9 \mathrm{mmHg})$ reduction in SBP and DBP, respectively over a five-week study period, thus confirming that dietary supplementation with $2 \mathrm{~g}$ of DHA per day lowered BP in persons with stable, untreated hypertension. In hypertensive patients, a modest BP lowering effect has been shown after fish oil intake in some [18, 19] but not all [20] studies. As previously shown [9], the greatest decrease in $\mathrm{BP}$ occurred in those persons with the lowest baseline plasma concentrations of DHA. This may indicate that an initially high acceptor-pool for PUFAs is important for the replacement of $\omega-6$ PUFAs by $\omega-3$ PUFAs during fish oil intake. Incorporation of $\omega-3$ PUFAs in biomembranes may favor the production of less vasoconstrictive thromboxane $\mathrm{A}_{3}$ rather than the arachidonic acid-derived thromboxane $\mathrm{A}_{2}$ [21]. Or $\omega-3$ PUFAs may interfere with the arterial baroreceptor of vascular resistance [22] and may thereby contribute to the reduction in BP.

Our finding that DHA had no effect on TC or non-HDL$\mathrm{C}$ is consistent with the findings by several other studies. Eslick et al. performed a meta-analysis of randomized trials of hyperlipidemic subjects and concluded that fish oil is effective in reducing triglycerides levels and had no effect on TC, although there were minor effects on HDL and LDL cholesterol [23]. Holub also reported in a review article that the benefits recognized for DHA on risk factors for CVD are independent of blood cholesterol lowering [24], and fasting plasma (serum) triglycerides are an important and independent risk factor for CHD [25].

The present results demonstrated a significant increase in HDL-C of $16.7 \%(8.6 \mathrm{mg} / \mathrm{dL})$ in DHA group. This is consistent with the result reported by Eslick and Holub that DHA supplementation has a moderate effect of increasing HDL cholesterol. In this study, a DHA dose of $2 \mathrm{~g}$ per day was sufficient to provide beneficial effects (HDL-C elevation) without clinically important adverse changes on TC and 


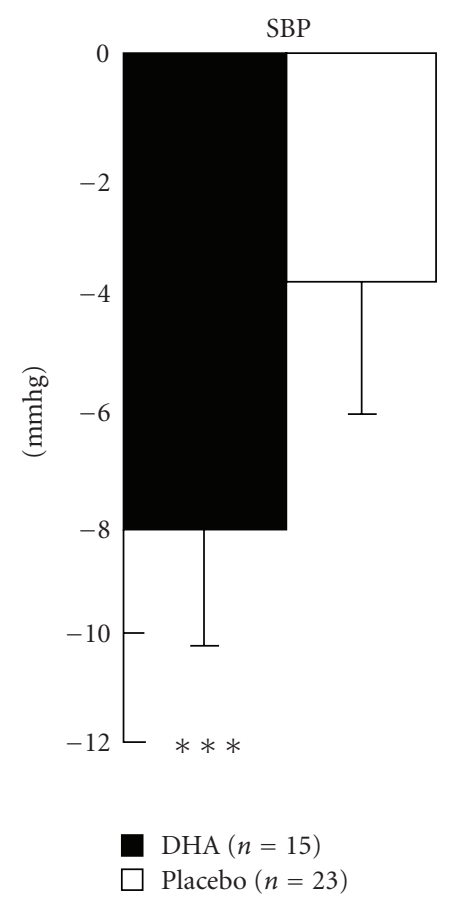

(a)

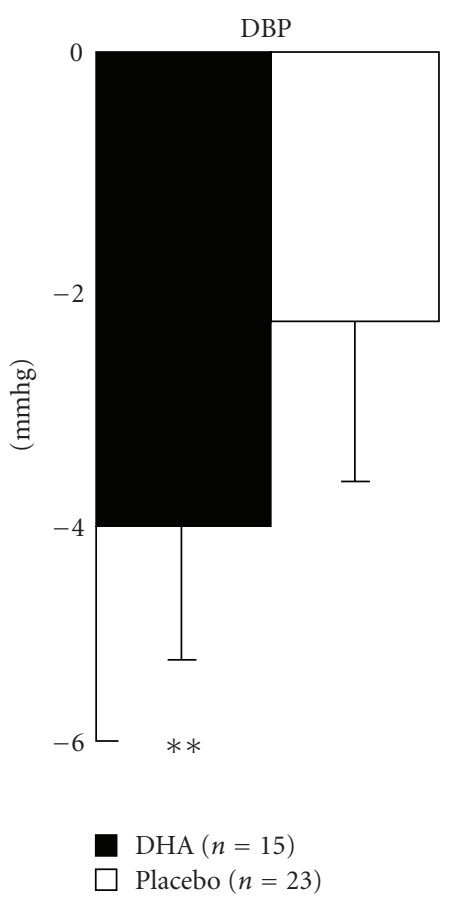

(b)

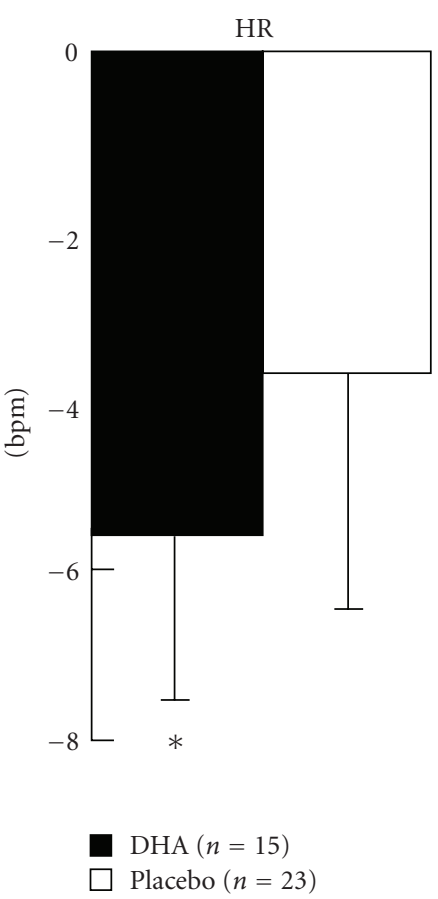

(c)

FIGURE 1: Effects of the DHA and placebo on systolic blood pressure (SBP), diastolic blood pressure (DBP), and heart rate (HR). Placebo: Olive Oil-containing bread rolls, DHA: DHA-containing bread rolls. Values are shown as mean \pm S.E. of the changes between baseline and after intervention. ${ }^{*} P<.05,{ }^{* *} P<.01$, and ${ }^{* * *} P<.001$ compared with the baselines. There was no significant difference between the two groups in either parameter.

non-HDL-C. That the ratios TC/HDL-C and non-HDLC/HDL-C, which are predictors of CHD risk, [16] decreased (therefore improved) significantly in both groups, would appear to be attributable to this. The increase in HDL-C with DHA supplementation may be mediated through a reduction in cholesteryl ester-triglyceride exchange between HDL-C and very low-density lipoprotein cholesterol particles as a consequence of triglyceride lowering. Harris has shown that triglyceride lowering effects of $\omega$-3 PUFAs appear to be due to a decrease in the production of hepatic triglycerides and an increase in the clearance of plasma triglycerides [26].

The placebo group also demonstrated a significant increase in HDL-C of $9.9 \%(4.5 \mathrm{mg} / \mathrm{dL})$ and decrease in the ratios of TC/HDL-C and non-HDL-C/HDL-C by $8.3 \%$ $(-0.5)$ and $10.2 \%(-0.5)$, respectively. These changes would be attributed to the effect of active placebo containing $1 \mathrm{~g} /$ day of olive oil. The results of this study strongly support the reported beneficial effect of olive oil, in that it appears to improve lipid profiles, that is, it increases serum HDL-C while decreasing triglycerides and the TC/HDL cholesterol ratio [27]. This further emphasizes the importance of routinely monitoring the effects of intervention strategies using any of these ratios.

It has been reported in the Framingham Study that elevated HR may be an independent risk factor for cardiovascular death in persons with hypertension [28]. There has also been data of non-Western populations. NIPPONDATA80 study showed that high resting $\mathrm{HR}$ is an independent predictor of cardiovascular and all-cause death for middle-aged men and women in the Japanese general population [29]. Mozaffarian reported in a meta-analysis of randomized controlled trials that fish oil reduces $H R$, particularly in those with higher baseline HR or longer treatment duration, and that these findings provide firm evidence that fish oil consumption directly or indirectly affects cardiac electrophysiology in humans [11]. Considering these reports together, our present result that HR was reduced by $7.5 \%$ $(-5.6 \mathrm{bpm})$ in DHA group may explain the effect of fish oil that reduces the risks of CVD. Previous CARDIAC largepopulation-based analysis also indicated that HR reduction is significantly associated with BP reduction, possibly contributing to CVD risk reduction [30]. The mechanism of $\mathrm{HR}$ reduction by fish oil is less certain but some possibilities are discussed. $\omega$-3 PUFAs incorporated into cardiomyocyte phospholipids [31] may alter ion channel function such as the modulation of the fast voltage-dependant $\mathrm{Na}^{+}$currents [32] and the L-type $\mathrm{Ca}^{2+}$ channels [33]. This would affect sinus node automaticity or responsiveness. Another possible mechanism is that by reducing systemic vascular resistance [34], fish oil would reduce $\mathrm{BP}[18,19]$ and improve ventricular efficiency, and this would induce a reduction of HR.

Subject compliance with the study protocol was determined to be high on the basis of record of counts of leftover bread rolls and the analysis of the FAs composition in plasma phospholipids. The latter has been shown to be a suitable biological marker for dietary $\omega-3$ PUFAs (DHA and EPA) intake [35]. 
There were some limitations to our study. Firstly, we had to use, as an active placebo, olive oil which was supposed to be good for health because of the ethical consideration of the study design. Therefore, it was difficult to obtain betweengroup significant differences. Secondly, this study is lacking in some data representing lipid profiles. Triglycerides were not assayed because it was susceptible to the diet and it was difficult to ask the participants to fast for more than 12 hours. LDL-cholesterol was not analyzed because frozen serum samples should be sent for the standardized analytical center in accordance with the WHO-CARDIAC multicenter study protocol [15]. Apolipoproteins which have received attention in recent years as CHD risk factors [36] were not analysed because it was difficult to set up a standardized method of analysis at the time when WHO-CARDIAC Study was designed in 1985 [15]. Thirdly, the sample size was small because this report was focused on a part of the intervention study designed to be carried out depending on the baseline data of different high risk populations examined by WHOCARDIAC Study.

However, some strengths were also present. Olive oil and fish oil, two main sources of fat in the Mediterranean diet, well known for the beneficial influence on CVD risks, were both effective for improving lipid profile, and DHA contained in fish oil demonstrated a greater effect on BP and HR than did olive oil within a limited time period.

\section{Conclusion}

In conclusion, supplementation with fish-oil-derived DHA incorporated into bread rolls reduced BP, HR, TC/HDL-C, and non-HDL-C/HDL-C ratios and increased HDL-C significantly, while did not affect TC or non-HDL-C in men with hypertension and/or hyper-cholesterolemia in the Isle of Lewis and Harris, where the fish intake is relatively low. These results indicate that the supplementation of DHA in daily bread is effective in reducing the risk factors of CHD even in those who do not eat fish frequently. Still further research is warranted to more clearly define the effects of DHA supplementation on lipid metabolism and cardiovascular risk.

\section{Acknowledgments}

The authors greatly acknowledge the Chief Executive and staff of the Western Isles Hospital in Stornoway as well as the local health centres in the isle of Lewis and Harris for their incredible technical and general support during the study period thus making it a success. They extend their most sincere appreciation to the study participants and their families and commend them for their dedication, motivation, and sincere interest in the research project, without whom these important findings among the Scottish population would have not been known. Their thanks are also extended to Dr. Kiyoshi Kumabe, President, KITII Co. Tokyo, Japan for his generous supply of calcium-encapsulated DHA and olive oil powder. Funding was provided by International Center for Primary Prevention of Cardiovascular Disease.

\section{References}

[1] M. G. Marmot, "Life style and national and international trends in coronary heart disease mortality," Postgraduate Medical Journal, vol. 60, no. 699, pp. 3-8, 1984.

[2] Scottish Public Health Observatory, Scotland and European Health for All Database 2009.

[3] Y. Yamori, Y. Nara, K. Ikeda, and S. Mizushima, "Is taurine a preventive nutritional factor of cardiovascular diseases or just a biological marker of nutrition?" Advances in Experimental Medicine and Biology, vol. 403, pp. 623-629, 1996.

[4] Y. Yamori, L. Liu, K. Ikeda et al., "Distribution of twentyfour hour urinary taurine excretion and association with ischemic heart disease mortality in 24 populations of 16 countries: results from the WHO-CARDIAC Study," Hypertension Research, vol. 24, no. 4, pp. 453-457, 2001.

[5] C. Bolton-Smith, W. C. S. Smith, M. Woodward, and H. Tunstall-Pedoe, "Nutrient intakes of different social-class groups: results from the Scottish Heart Health Study (SHHS)," British Journal of Nutrition, vol. 65, no. 3, pp. 321-335, 1991.

[6] Y. Kagawa, M. Nishizawa, M. Suzuki et al., "Eicosapolyenoic acids of serum lipids of Japanese islanders with low incidence of cardiovascular diseases," Journal of Nutritional Science and Vitaminology, vol. 28, no. 4, pp. 441-453, 1982.

[7] E. Dewailly, C. Blanchet, S. Lemieux et al., "n-3 Fatty acids and cardiovascular disease risk factors among the Inuit of Nunavik," American Journal of Clinical Nutrition, vol. 74, no. 4, pp. 464-473, 2001.

[8] S. Mizushima, E. H. Moriguchi, Y. Nakada et al., "The relationship of dietary factors to cardiovascular diseases among Japanese in Okinawa and Japanese immigrants, originally from Okinawa, in Brazil," Hypertension Research, vol. 15, pp. 45-55, 1992.

[9] K. H. Bonaa, K. S. Bjerve, B. Straume, I. T. Gram, and D. Thelle, "Effect of eicosapentaenoic and docosahexaenoic acids on blood pressure in hypertension. A population-based intervention trial from the Tromso study," The New England Journal of Medicine, vol. 322, no. 12, pp. 798-801, 1990.

[10] P. Weber and D. Raederstorff, "Triglyceride-lowering effect of omega-3 LC-polyunsaturated fatty acids-a review," Nutrition, Metabolism and Cardiovascular Diseases, vol. 10, no. 1, pp. 28-37, 2000.

[11] D. Mozaffarian, A. Geelen, I. A. Brouwer, J. M. Geleijnse, P. L. Zock, and M. B. Katan, "Effect of fish oil on heart rate in humans: a meta-analysis of randomized controlled trials," Circulation, vol. 112, no. 13, pp. 1945-1952, 2005.

[12] Y. Yamori, L. Liu, S. Mizushima, K. Ikeda, and Y. Nara, "Male cardiovascular mortality and dietary markers in 25 population samples of 16 countries," Journal of Hypertension, vol. 24, no. 8, pp. 1499-1505, 2006.

[13] R. A. Harrison, M. Sagara, A. Rajpura et al., "Can foods with added soya-protein or fish-oil reduce risk factors for coronary disease? A factorial randomised controlled trial," Nutrition, Metabolism and Cardiovascular Diseases, vol. 14, no. 6, pp. 344-350, 2004.

[14] M. Sagara, T. Kanda, M. Njelekera et al., "Effects of dietary intake of soy protein and isoflavones on cardiovascular disease risk factors in high risk, middle-aged men in Scotland," Journal of the American College of Nutrition, vol. 23, no. 1, pp. 85-91, 2004.

[15] Cardiovascular Diseases and Alimentary Comparison Study Protocol and Manual of Operations, "WHO Collaborating Center on Primary Prevention of Cardiovascular Disease, 
Izumo, Japan and Cardiovascular Diseases Unit," WHO, Geneva, Switzerland, pp. 56-58, 1986.

[16] Y. Cui, R. S. Blumenthal, J. A. Flaws et al., "Non-high-density lipoprotein cholesterol level as a predictor of cardiovascular disease mortality," Archives of Internal Medicine, vol. 161, no. 11, pp. 1413-1419, 2001.

[17] J. Folch, M. Lees, and S. Stanley, "A simple method for the isolation and purification of total lipides from animal tissues," The Journal of biological chemistry, vol. 226, no. 1, pp. 497-509, 1957.

[18] H. R. Knapp and G. A. Fitzgerald, "The antihypertensive effects of fish oil. A controlled study of polyunsaturated fatty acid supplements in essential hypertension," The New England Journal of Medicine, vol. 320, no. 16, pp. 1037-1043, 1989.

[19] I. Toft, K. H. Bonaa, O. C. Ingebretsen, A. Nordoy, and T. Jenssen, "Effects of n-3 polyunsaturated fatty acids on glucose homeostasis and blood pressure in essential hypertension. A randomized, controlled trial," Annals of Internal Medicine, vol. 123, no. 12, pp. 911-918, 1995.

[20] L. M. H. Wing, P. J. Nestel, J. P. Chalmers et al., "Lack of effect of fish oil supplementation on blood pressure in treated hypertensives," Journal of Hypertension, vol. 8, no. 4, pp. 339343, 1990.

[21] J. Dyerberg, H. O. Bang, and E. Stoffersen, "Eicosapentaenoic acid and prevention of thrombosis and atherosclerosis?" Lancet, vol. 2, no. 8081, pp. 117-119, 1978.

[22] B. Weisser, A. Struck, B. O. Gobel, H. Vetter, and R. Dusing, "Fish oil and baroreceptor function in man," Klinische Wochenschrift, vol. 68, supplement, pp. 49-52, 1990.

[23] G. D. Eslick, P. R. C. Howe, C. Smith, R. Priest, and A. Bensoussan, "Benefits of fish oil supplementation in hyperlipidemia: a systematic review and meta-analysis," International Journal of Cardiology, vol. 136, no. 1, pp. 4-16, 2009.

[24] B. J. Holub, "Docosahexaenoic acid (DHA) and cardiovascular disease risk factors," Prostaglandins Leukotrienes and Essential Fatty Acids, vol. 81, no. 2-3, pp. 199-204, 2009.

[25] A. M. Gotto, “Triglyceride: the forgotten risk factor," Circulation, vol. 97, no. 11, pp. 1027-1028, 1998.

[26] W. S. Harris, M. Miller, A. P. Tighe, M. H. Davidson, and E. J. Schaefer, "Omega-3 fatty acids and coronary heart disease risk: clinical and mechanistic perspectives," Atherosclerosis, vol. 197, no. 1, pp. 12-24, 2008.

[27] M. Fito, R. De La Torre, M. Farré-Albaladejo, O. Khymenetz, J. Marrugat, and M. I. Covas, "Bioavailability and antioxidant effects of olive oil phenolic compounds in humans: a review," Annali dell'Istituto Superiore di Sanita, vol. 43, no. 4, pp. 375381, 2007.

[28] M. W. Gillman, W. B. Kannel, A. Belanger, and R. B. D'Agostino, "Influence of heart rate on mortality among persons with hypertension: the Framingham Study," American Heart Journal, vol. 125, no. 4, pp. 1148-1154, 1993.

[29] T. Okamura, T. Hayakawa, T. Kadowaki et al., "Resting heart rate and cause-specific death in a 16.5-year cohort study of the Japanese general population," American Heart Journal, vol. 147, no. 6, pp. 1024-1032, 2004.

[30] L. Liu, S. Mizushima, K. Ikeda, Y. Nara, and Y. Yamori, "Resting heart rate in relation to blood pressure: results from the World Health Organization-Cardiovascular Disease and Alimentary Comparison Study," International Journal of Cardiology, vol. 145, no. 1, pp. 73-74, 2010.

[31] R. G. Metcalf, M. J. James, R. A. Gibson et al., "Effects of fishoil supplementation on myocardial fatty acids in humans," American Journal of Clinical Nutrition, vol. 85, no. 5, pp. 1222 1228, 2007.
[32] A. Leaf, J. X. Kang, Y. F. Xiao, and G. E. Billman, "Clinical prevention of sudden cardiac death by $n-3$ polyunsaturated fatty acids and mechanism of prevention of arrhythmias by n3 fish oils," Circulation, vol. 107, no. 21, pp. 2646-2652, 2003.

[33] H. Hallaq, T. W. Smith, and A. Leaf, "Modulation of dihydropyridine-sensitive calcium channels in heart cells by fish oil fatty acids," Proceedings of the National Academy of Sciences of the United States of America, vol. 89, no. 5, pp. 17601764, 1992.

[34] D. Mozaffarian, J. S. Gottdiener, and D. S. Siscovick, "Intake of tuna or other broiled or baked fish versus fried fish and cardiac structure, function, and hemodynamics," American Journal of Cardiology, vol. 97, no. 2, pp. 216-222, 2006.

[35] K. S. Bjerve, A. M. Brubakk, K. J. Fougner et al., "Omega3 fatty acids: essential fatty acids with important biological effects, and serum phospholipid fatty acids as markers of dietary $\omega 3$-fatty acid intake," American Journal of Clinical Nutrition, vol. 57, supplement, pp. S801-S806, 1993.

[36] M. J. McQueen, S. Hawken, X. Wang et al., "Lipids, lipoproteins, and apolipoproteins as risk markers of myocardial infarction in 52 countries (the INTERHEART study): a casecontrol study," The Lancet, vol. 372, no. 9634, pp. 224-233, 2008 . 


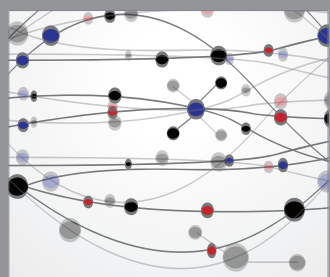

The Scientific World Journal
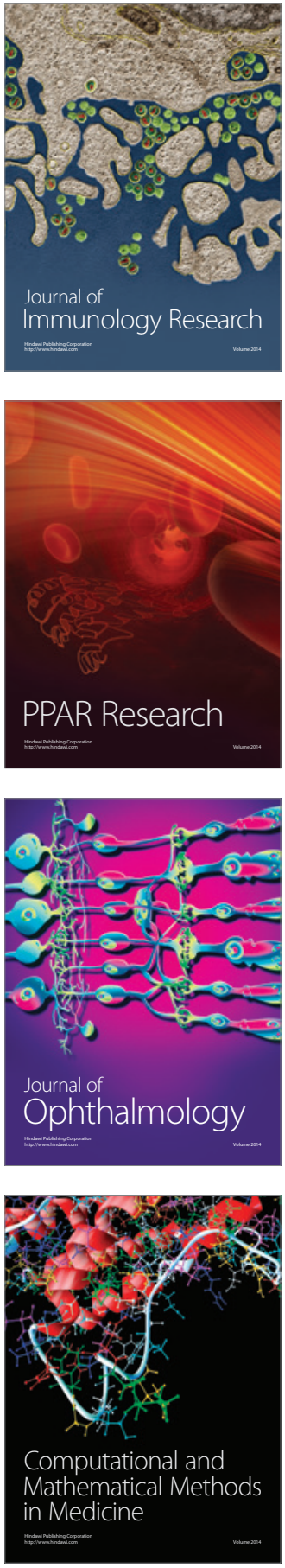

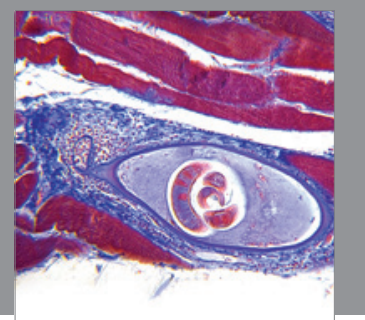

Gastroenterology

Research and Practice
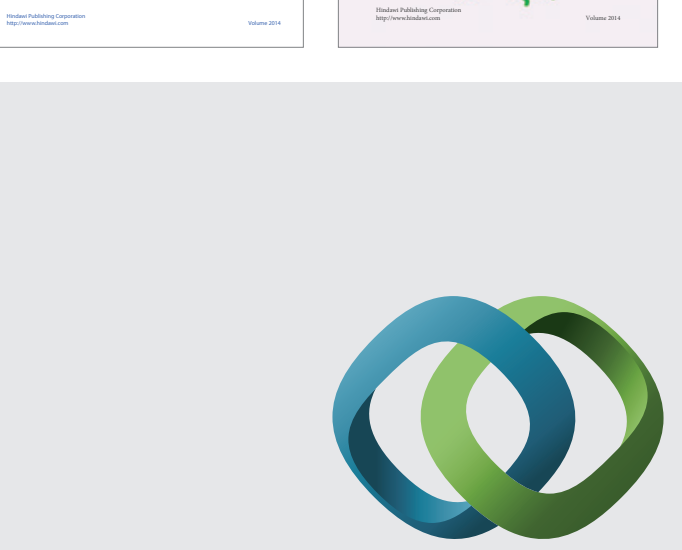

\section{Hindawi}

Submit your manuscripts at

http://www.hindawi.com
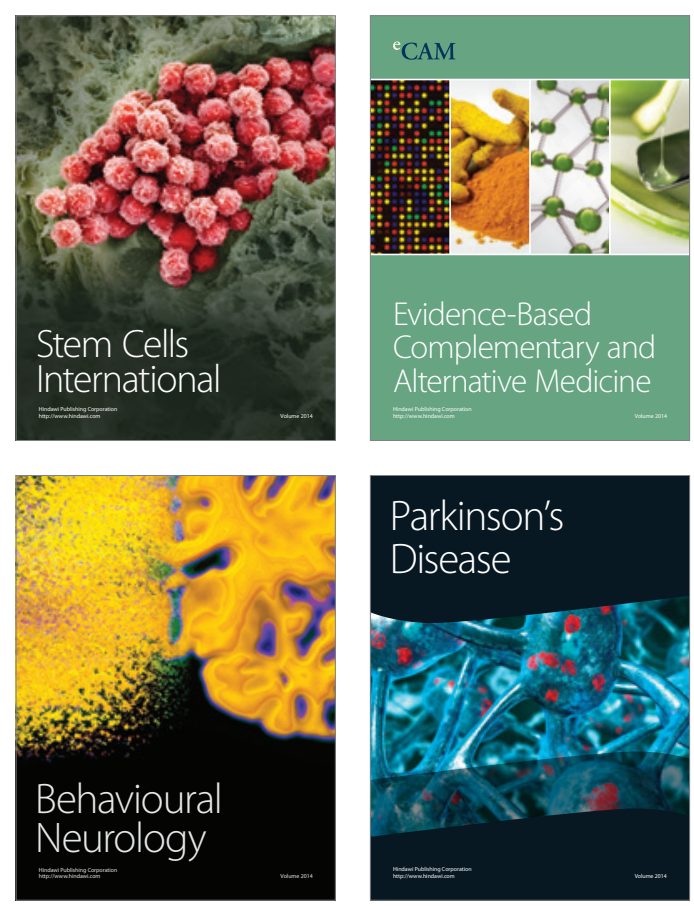

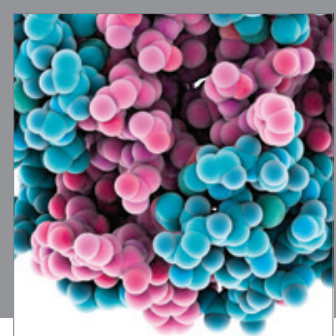

Journal of
Diabetes Research

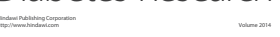

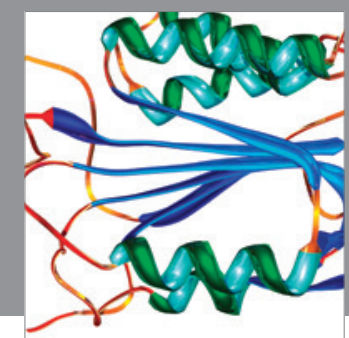

Disease Markers
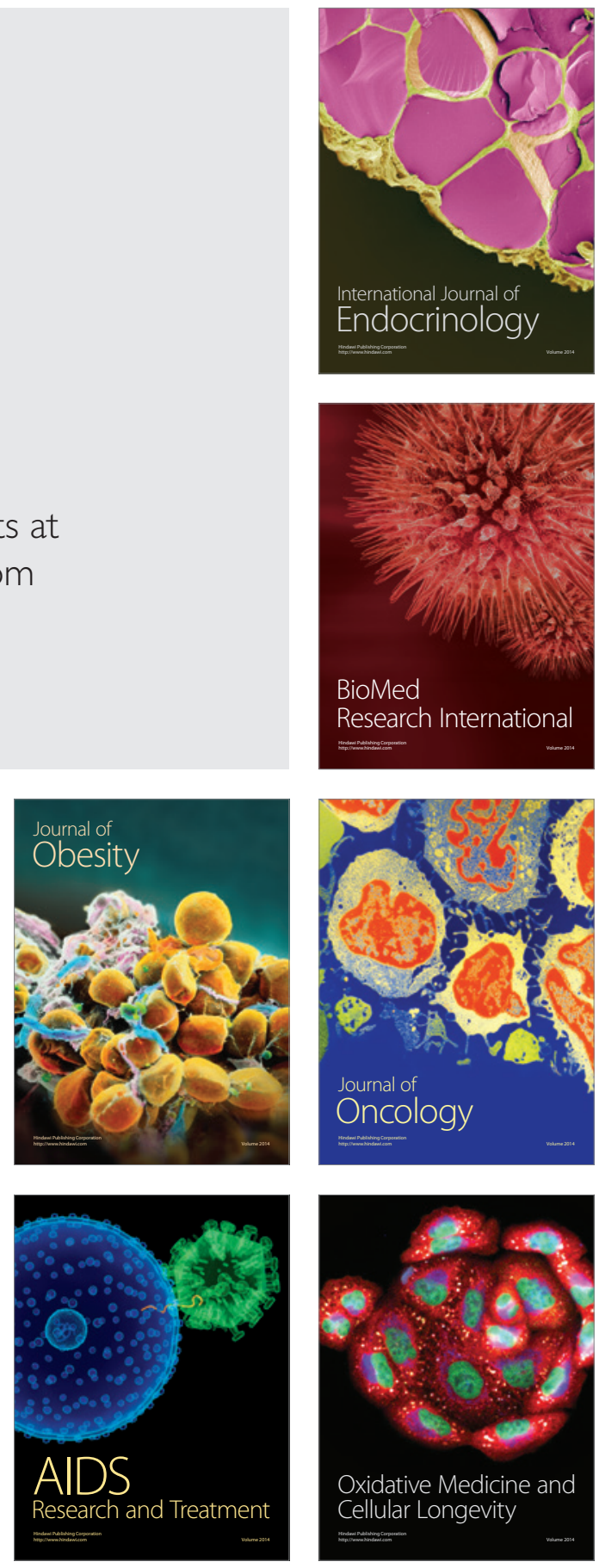